\title{
B cells control lupus autoimmunity by inhibiting Th17 and promoting Th22 cells
}

\author{
Ji Yang ${ }^{1}$, Xue Yang ${ }^{2,3}$, Luman Wang ${ }^{4}$ and Ming Li ${ }^{1}$
}

\begin{abstract}
B cells exert immunosuppressive effects and offer therapeutic potential for systemic lupus erythematosus (SLE), but the mechanism remains unclear. Here we analyzed the B cell regulation of Th17/Th22 cell differentiation in lupus and found that a-IgM- and a-CD40-activated B cells could inhibit Th17 and promote Th22 cell differentiation from naive T cells under Th17 cell culture conditions. B cell-induced Th22 cells demonstrated immunosuppressive effects and could decrease renal endothelial cell apoptosis in vitro. Moreover, activated B cell infusion relieved lupus injuries via IL22 production in vivo. Mechanically, activated B cells affected Th17/Th22 cell differentiation by non-contact TNF-a secretion and mTOR activation. Finally, activated B cells could affect Th17/Th22 cell differentiation in human peripheral blood T cells. These data suggest that activated B cells might attenuate lupus autoimmunity by inhibiting Th17 but promoting Th22 cell differentiation, supporting B cell activation as a promising therapeutic for the treatment of lupus.
\end{abstract}

\section{Introduction}

Systemic lupus erythematosus (SLE) is a common autoimmune disease that involves multiple organ systems, occurring in $20-150$ for every 100,000 people $^{1}$. The pathogenesis of SLE remains unclear, although an imbalance within the immune system has been implicated. Therefore, it is necessary to investigate the immunological mechanisms of lupus to provide greater clarity and knowledge toward viable treatments.

In the disease microenvironment, B cells can be activated and induced into regulatory immune cells that affect disease progression ${ }^{2-5}$. Activated B cells produce cytokines such as interleukin (IL)-10, transforming growth factor (TGF)- $\beta$, and tumor necrosis factor (TNF)- $\alpha^{6,7}$, and the absence of $B$ cells exacerbated disease symptoms in models of lupus, experimental autoimmune encephalomyelitis, and collagen-induced arthritis ${ }^{3,4,8-10}$. Previous

\footnotetext{
Correspondence: Ji Yang (yang.ji@zs-hospital.sh.cn)

'Department of Dermatology, Zhongshan Hospital, Fudan University, Shanghai, China

Division of Rheumatology, Huashan Hospital, Fudan University, Shanghai, China

Full list of author information is available at the end of the article. These authors contributed equally: Ji Yang, Xue Yang, Luman Wang Edited by H.U. Simon
}

studies have shown B cell dysregulation in SLE patients and MRL/lpr mice ${ }^{10-12}$, especially with immunosuppressive functions ${ }^{13}$. These immunosuppressive effects could exert therapeutic benefits against lupus ${ }^{14}$, but how B cells are regulated is not fully understood.

The T helper 17 (Th17) cell lineage, a lineage of effector $\mathrm{CD}^{+} \mathrm{T}$ cells characterized by IL-17 production ${ }^{15,16}$, is associated with the pathogenesis of autoimmune diseases, including $\mathrm{SLE}^{17-20}$. Our studies, as well as others, have shown that Th17 cells were expanded in SLE similar to inflammatory tissue injuries and autoantibody production $^{17,20,21}$. Thus Th17 cell inhibition could help to relieve lupus autoimmune injuries. IL-22-producing $\mathrm{CD}^{+} \mathrm{T}$ (Th22) cells are a new subset of $\mathrm{CD} 4^{+} \mathrm{T}$ cells with immunosuppressive capabilities and differentiated from naive $\mathrm{T}$ cells through TNF- $\alpha$ and IL- $6^{22,23}$. Although IL22 can be produced during Th17 cell differentiation ${ }^{24,25}$, the reciprocal differentiation of Th17 by Th22 cells in lupus autoimmunity is not clear.

Because the potential for B cells to affect Th17 and Th22 cell differentiation has not been reported, we have analyzed the mechanisms and potential therapeutic role through which B cells affect Th17 and Th22 cell differentiation in the treatment of lupus. 


\section{Materials and methods Mice treatment}

CD45.2 ${ }^{+}$C57BL/6 (B6) and lupus-prone MRL/lpr mice were purchased from the Shanghai Laboratory Animal Center (Chinese Academy of Sciences). CD45.1 ${ }^{+}$mice were purchased from The Jackson Laboratory (Bar Harbor, ME). Animal studies were approved by the Institutional Animal Care and Use Committee of Zhongshan Hospital, Fudan University. Mice were maintained under pathogen-free conditions. Twelve-week-old MRL/lpr mice were randomized into four groups, and the mice were injected intravenously with $10 \times 10^{6}$ ex vivoexpanded B cells or phosphate-buffered saline (PBS) control with or without $2.5 \mu \mathrm{g} / \mathrm{g}$ anti-IL-22 antibody (Thermo Fisher Scientific, Waltham, MA, USA) weekly for 4 weeks. The animal study is not blinding. Urine was collected for the first $24 \mathrm{~h}$ and assayed to detect protein by Coomassie brilliant blue according to the manufacturer's instructions (Nanjing Jiancheng, China). Four weeks after treatment, MRL/lpr mice were sacrificed and the spleens and inguinal lymph nodes were collected and weighed. The percentages of $\mathrm{CD} 4^{+} \mathrm{IL}-17^{+}$Th17 cells and CD $4^{+} \mathrm{IL}-$ $22^{+}$Th22 cells in the spleens were analyzed by flow cytometry, including retinoic acid-related orphan receptor $\gamma \mathrm{t}$ (ROR $\gamma \mathrm{t}$ ) and $\mathrm{c}$-Maf intracellular expression. Kidney tissues were fixed for assessment.

\section{Naive $\mathrm{CD}^{+}{ }^{+} \mathrm{T}$ and $\mathrm{B}$ cell isolation and differentiation}

For Th17 cell differentiation, naive $\mathrm{CD} 4^{+} \mathrm{T}$ cells were purified from the spleens of B6 mice using the naive Mouse CD4 Cell Kit (StemCell Technologies, Vancouver, $\mathrm{BC}$, Canada). Sorted naive $\mathrm{CD} 4^{+} \mathrm{T}$ cells were cultured under Th17 cell culture conditions with $2 \mu \mathrm{g} / \mathrm{mL}$ antiCD3, $2 \mu \mathrm{g} / \mathrm{mL}$ anti-CD28, $1 \mathrm{ng} / \mathrm{mL}$ TGF- $\beta, 50 \mathrm{ng} / \mathrm{mL}$ IL6, $10 \mathrm{ng} / \mathrm{mL}$ IL-1 $\beta, 5 \mathrm{ng} / \mathrm{mL}$ IL-23, $10 \mu \mathrm{g} / \mathrm{mL}$ anti-IL-4, $10 \mu \mathrm{g} / \mathrm{mL}$ anti-IFN- $\gamma$, and $10 \mu \mathrm{g} / \mathrm{mL}$ anti-IL-2 (all from Thermo Fisher Scientific) for 5 days.

For Th1 cell differentiation, naive $\mathrm{CD}^{+} \mathrm{T}$ cells were purified from the spleens of B6 mice using the naive Mouse CD4 Cell Kit (StemCell Technologies, Vancouver, $\mathrm{BC}$, Canada). Sorted naive $\mathrm{CD}^{+} \mathrm{T}$ cells were cultured under Th1 cell culture conditions with $2 \mu \mathrm{g} / \mathrm{mL}$ anti-CD3, $2 \mu \mathrm{g} / \mathrm{mL}$ anti-CD28, $20 \mathrm{ng} / \mathrm{mL}$ IL-2, $20 \mathrm{ng} / \mathrm{mL}$ IL-12, and $10 \mu \mathrm{g} / \mathrm{mL}$ anti-IL-4 (all from Thermo Fisher Scientific) for 5 days.

For regulatory $\mathrm{T}$ (Treg) cell differentiation, naive $\mathrm{CD}^{+}$ $\mathrm{T}$ cells were purified from the spleens of B6 mice using the naive Mouse CD4 Cell Kit (StemCell Technologies, Vancouver, BC, Canada). Sorted naive CD4 ${ }^{+} \mathrm{T}$ cells were cultured under Treg cell culture conditions with $2 \mu \mathrm{g} / \mathrm{mL}$ anti-CD3, $2 \mu \mathrm{g} / \mathrm{mL}$ anti-CD28, $5 \mathrm{ng} / \mathrm{mL}$ IL-2, and $5 \mathrm{ng} /$ $\mathrm{mL}$ recombinant human TGF- $\beta 1$ for 5 days.

For B cell differentiation, naive B cells were purified from the spleens of B6 mice using the Mouse B Cell Kit
(StemCell Technologies). Sorted naive B cells were stimulated for 2 days with $2 \mu \mathrm{g} / \mathrm{mL}$ anti-CD40L and $2 \mu \mathrm{g} /$ mL anti-IgM (BD Pharmingen, San Diego, CA, USA).

For some experiments, sorted naive $B$ cells were stimulated with $2 \mu \mathrm{g} / \mathrm{mL}$ anti-CD40L and $2 \mu \mathrm{g} / \mathrm{mL}$ anti-IgM (BD Pharmingen) for 2 days and then co-cultured with naive $\mathrm{T}$ cells under $\mathrm{Th} 17$ cell culture conditions for 5 days. For some experiments, induced B cells were cocultured with pre-Th17 cells (naive T cells stimulated in Th17 culture conditions for 3 days) for an additional 5 days, after which Th17 and Th22 cell differentiation was analyzed.

For some experiments, sorted naive B cells were stimulated with $2 \mu \mathrm{g} / \mathrm{mL}$ anti-CD40L and $2 \mu \mathrm{g} / \mathrm{mL}$ anti-IgM (BD Pharmingen) for 2 days and then co-cultured with naive $\mathrm{T}$ cells under Th1 cell culture condition or Treg for 5 days.

For some experiments, sorted naive B cells were stimulated with $2 \mu \mathrm{g} / \mathrm{mL}$ anti-CD40L and $2 \mu \mathrm{g} / \mathrm{mL}$ anti-IgM (BD Pharmingen) for 2 days, then co-cultured with naive $\mathrm{T}$ cells in different transwell chambers under Th17 cell culture conditions with or without $1 \mu \mathrm{g} / \mathrm{mL}$ anti-TNF- $\alpha$ antibody or $5 \mathrm{ng} / \mathrm{mL}$ TNF- $\alpha$ (Thermo Fisher Scientific) for 5 days, after which Th17 and Th22 cell differentiation was analyzed.

For some experiments, naive $B$ cells sorted from CD $45.1^{+}$mice were stimulated with $2 \mu \mathrm{g} / \mathrm{mL}$ anti-CD40L and $2 \mu \mathrm{g} / \mathrm{mL}$ anti-IgM (BD Pharmingen) for 2 days, then co-cultured with naive $\mathrm{T}$ cells sorted from CD45.1 ${ }^{+}$mice under Th17 cell culture conditions for 5 days. These induced CD45.1 $1^{+} \mathrm{T}$ cells were sorted and co-cultured with primary renal endothelial cells or naive $\mathrm{T}$ or $\mathrm{B}$ cells sorted from CD45.2 $2^{+}$mice for 3 days under different culture conditions. Renal endothelial cell apoptosis was analyzed by flow cytometry, and $\mathrm{T}$ and $\mathrm{B}$ cell differentiation was analyzed.

For some experiments, sorted naive B cells were stimulated with $2 \mu \mathrm{g} / \mathrm{mL}$ anti-CD40L and $2 \mu \mathrm{g} / \mathrm{mL}$ anti-IgM (BD Pharmingen) for 2 days, then co-cultured with naive $\mathrm{T}$ cells under Th17 cell culture conditions with or without $1 \mu \mathrm{g} / \mathrm{mL}$ anti-TNF- $\alpha$ antibody, $5 \mathrm{ng} / \mathrm{mL}$ TNF- $\alpha$ (Thermo Fisher Scientific), $10 \mu \mathrm{M}$ mammalian target of rapamycin (mTOR) agonist (MHY1485; MedChem Express, USA), or $200 \mathrm{ng} / \mathrm{mL}$ rapamycin. mTOR phosphorylation and IL17 and IL-22 production were then analyzed.

For some experiments, sorted naive B cells from the peripheral blood mononuclear cells (PBMCs) of 3 healthy human donors were stimulated with $2 \mu \mathrm{g} / \mathrm{mL}$ anti-CD40L and $2 \mu \mathrm{g} / \mathrm{mL}$ anti-IgM (BD Pharmingen) for 2 days, then co-cultured with naive $\mathrm{T}$ cells sorted from healthy human donor PMBCs under Th17 cell culture conditions for 5 days. Th17 and Th22 cell differentiation was then analyzed. The study protocol was reviewed and approved by the ZhongShan Hospital Research Ethics Committee. 


\section{Flow cytometric analysis}

To detect Th17 and Th22 cells, cells were incubated for $5 \mathrm{~h}$ with $50 \mathrm{ng} / \mathrm{mL}$ phorbol myristate acetate (PMA; Sigma-Aldrich, USA) and $750 \mathrm{ng} / \mathrm{mL}$ ionomycin (SigmaAldrich) in the presence of $20 \mu \mathrm{g} / \mathrm{mL}$ brefeldin A (SigmaAldrich) and then stained with fluorescein isothiocyanate (FITC)-conjugated anti-CD4 for $15 \mathrm{~min}$. Cells were then resuspended in a fixation/permeabilization solution and stained intracellularly with phycoerythrin (PE)-conjugated anti-IL-17, PE-conjugated anti-IL-22, PE-conjugated antiRORyt, or PE-conjugated anti-c-Maf for 30 min according to the manufacturer's instructions (Thermo Fisher Scientific). After staining, $\mathrm{IL}-17^{+}, \mathrm{IL}-22^{+}, \mathrm{ROR}^{+}{ }^{+}$, and c$\mathrm{Maf}^{+}$cells were analyzed with a $\mathrm{CD}^{+}$gate by flow cytometry.

For Th1 cell-related cytokine detection, cells were incubated for $5 \mathrm{~h}$ with $50 \mathrm{ng} / \mathrm{mL}$ PMA and $750 \mathrm{ng} / \mathrm{mL}$ ionomycin in the presence of $20 \mu \mathrm{g} / \mathrm{mL}$ brefeldin $\mathrm{A}$, then stained with FITC-conjugated anti-CD4 for $15 \mathrm{~min}$. Cells were then resuspended in a fixation/permeabilization solution and stained intracellularly with PE-conjugated anti-TNF- $\alpha$, PE-conjugated anti-IFN- $\gamma$, or PE-conjugated anti-TGF- $\beta$ for $30 \mathrm{~min}$ according to the manufacturer's instructions (Thermo Fisher Scientific). After staining, TNF- $\alpha^{+}$, IFN $-\gamma^{+}$, and TGF- $\beta^{+}$cells were analyzed with a $\mathrm{CD} 4^{+}$gate by flow cytometry.

For IL-10 and TGF- $\beta$ detection in B cells, cells were incubated for $5 \mathrm{~h}$ with $50 \mathrm{ng} / \mathrm{mL}$ PMA and $750 \mathrm{ng} / \mathrm{mL}$ ionomycin in the presence of $20 \mu \mathrm{g} / \mathrm{mL}$ brefeldin $\mathrm{A}$, then surface-stained with FITC-conjugated anti-CD19 for $15 \mathrm{~min}$. Cells were then re-suspended in Fixation/Permeabilization solution (Invitrogen, USA) and stained intracellularly with PE-conjugated anti-IL-10 or PEconjugated anti-TGF- $\beta$ for $30 \mathrm{~min}$ according to the manufacturer's protocol (Thermo Fisher Scientific). After staining, IL- $10^{+}$and TGF- $\beta^{+}$cells were analyzed with a $\mathrm{CD} 19^{+}$gate by flow cytometry.

For renal endothelial cell apoptosis detection, C57BL/6 mouse primary kidney endothelial cells purchased from Cell Biologics (Chicago, IL, USA) were co-cultured with B cell-induced $\mathrm{CD} 45.1^{+} \mathrm{T}$ cells for 3 days. Then endothelial cell apoptosis was analyzed by the Annexin V Apoptosis Detection Kit (BD Pharmingen). The expression levels of IL-22R (Novus Biologicals, Littleton, CO, USA), CD31, CD45, and ICAM (eBioscience) were detected by flow cytometry.

\section{Cytokine detection}

Sorted T or B cells from mice were cultured with or without B cells, then IL-17, IL-22, TNF- $\alpha$, interferon (IFN)- $\gamma$, TGF- $\beta$, IL-10, immunoglobulin $M$ (IgM), and IgG levels in supernatants were determined by enzymelinked immunosorbent assay (ELISA; all from Thermo Fisher Scientific). Serum double-stranded DNA (ds-DNA) antibody levels in MRL/lpr mice were detected by ELISA (Thermo Fisher Scientific). Sorted $\mathrm{T}$ or B cells from healthy human donor PBMCs were cultured with or without B cells, then IL-17 and IL-22 levels in supernatants were determined by ELISA (all from Thermo Fisher Scientific).

\section{Histopathological assessment}

Mouse kidneys were fixed with formaldehyde, embedded in paraffin, and stained with hematoxylin and eosin (H\&E). H\&E-stained kidney slides were read and interpreted in a blind fashion in which kidneys were graded for glomerular inflammation, proliferation, crescent formation, and necrosis. Interstitial changes and vasculitis were also noted. Scores from 0 to 3 were assigned for each feature, and scores were added to yield a final renal score. For example, glomerular inflammation was graded as follows: 0 , normal; 1 , few inflammatory cells; 2 , moderate inflammation; and 3, severe inflammation.

\section{Statistical analysis}

Quantitative data were expressed as mean \pm standard deviation (SD). Differences were determined by unpaired two-tailed $t$ test for comparing two groups. For comparing two group values that did not follow Gaussian distribution, the two-tailed Mann-Whitney $U$ test was used. All $p$ values $<0.05$ were considered significant.

\section{Results}

\section{Activated B cells inhibit Th17 but promote Th22 cell differentiation in vitro}

B cells are potent negative regulators of inflammation and autoimmunity when activated in vivo and in vitro ${ }^{7,26}$. Here $\alpha$-IgM- and $\alpha$-CD40-activated B cells were cocultured with naive $\mathrm{T}$ cells under Th17 cell culture conditions. Compared with the $\mathrm{T}$ cell only group, activated $\mathrm{B}$ cells inhibited IL-17 production and RORyt expression (a transcription factor of Th17 cells ${ }^{27}$ ) (Fig. 1a-c). Interestingly, activated $\mathrm{B}$ cells promoted $\mathrm{CD} 4^{+} \mathrm{IL}-22^{+} \mathrm{T}$ cell differentiation and IL-22 secretion even under Th17 cell culture conditions (Fig. 1d, e). In addition, c-Maf, reported as a negative regulator of Th22 cell differentiation ${ }^{21}$, was inhibited by activated B cells (Fig. 1f). These data indicated that activated B cells could inhibit Th17 but promote Th22 cell differentiation. To further analyze whether B cells could reverse Th22 cell differentiation from Th17 cells, activated B cells were co-cultured with established Th17 cells (naive T cells pre-cultured under Th17 culture conditions for 3 days). B cells could neither affect the differentiation of Th17 and Th22 cells nor regulate RORyt and c-Maf expression (Fig. 1g-l). We also determined the effects of activated B cells on other effector $\mathrm{T}$ and Treg cell subsets. As Supplementary Fig. 1a-c shows, activated B cells did not affect Th1 or 


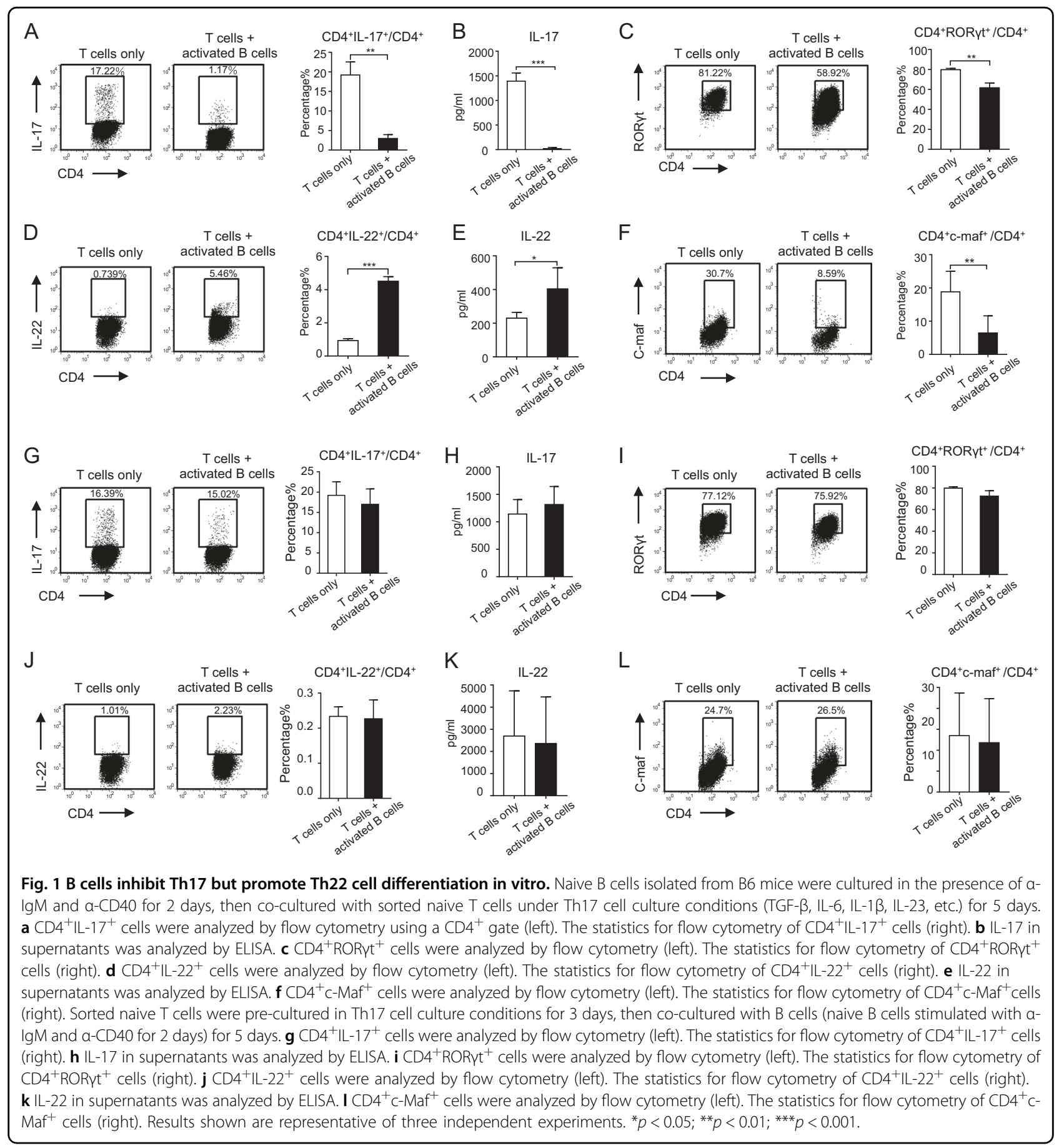

IL- $10^{+}$and TGF- $\beta^{+}$Treg cell differentiation. Collectively, these data indicate that activated $B$ cells might inhibit Th17 and promote Th22 cell differentiation from naive $\mathrm{T}$ cells but not differentiated Th17 cells.

\section{Activated B cell-induced Th22 cells display immunosuppressive effects in vitro}

To analyze the function of activated B cell-induced Th22 cells, induced Th22 cells from CD45.1 background mice were first sorted from the co-culture system on day 5 , then co-cultured with naive $\mathrm{T}$ and naive $\mathrm{B}$ cells sorted from CD45.2 background mice under different culture conditions (Fig. 2a, b). Activated $\mathrm{CD}^{+} \mathrm{T}$ cells had increased expression of IL-22R (blue line) compared with naive $\mathrm{T}$ cell (red line); B cells also had mildly increased expression of IL-22R (blue line) compared with naive $B$ cell (red line) (Fig. 2c). Induced Th22 cells did not affect T cell proliferation but significantly inhibited the TNF- $\alpha$ 


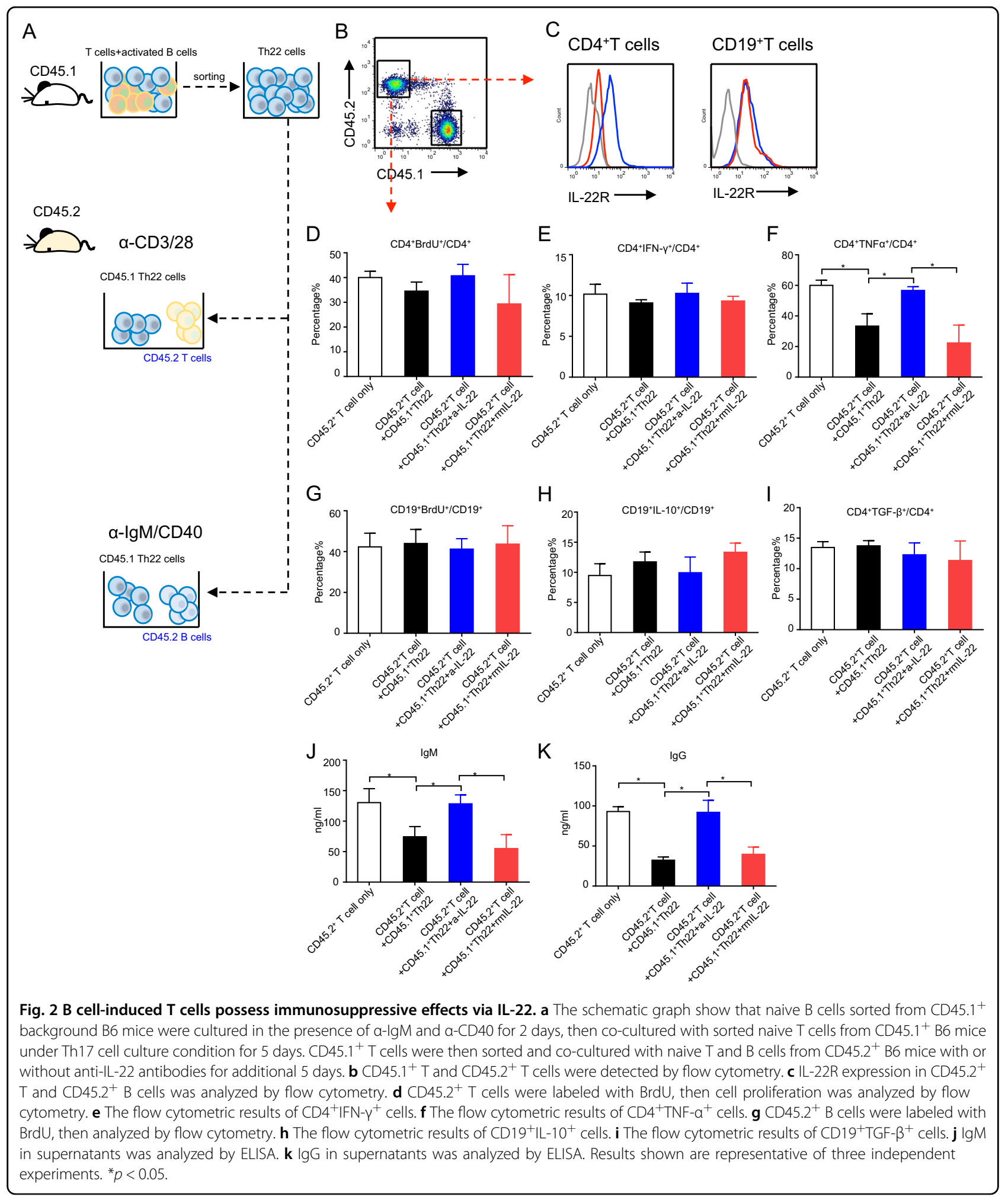

production of activated $\mathrm{T}$ cells (Fig. 2d-f). Moreover, induced Th22 cells did not affect $\mathrm{CD}_{19}{ }^{+} \mathrm{B}$ cell proliferation or IL-10 and TGF- $\beta$ production but inhibited IgM and IgG production (Fig. $2 \mathrm{~g}-\mathrm{k}$ ). These inhibitory effects could be reversed by supplementing culture media with anti-IL-22 antibody. The findings suggest that B cellinduced Th22 cells possess immunosuppressive effects via IL-22. 


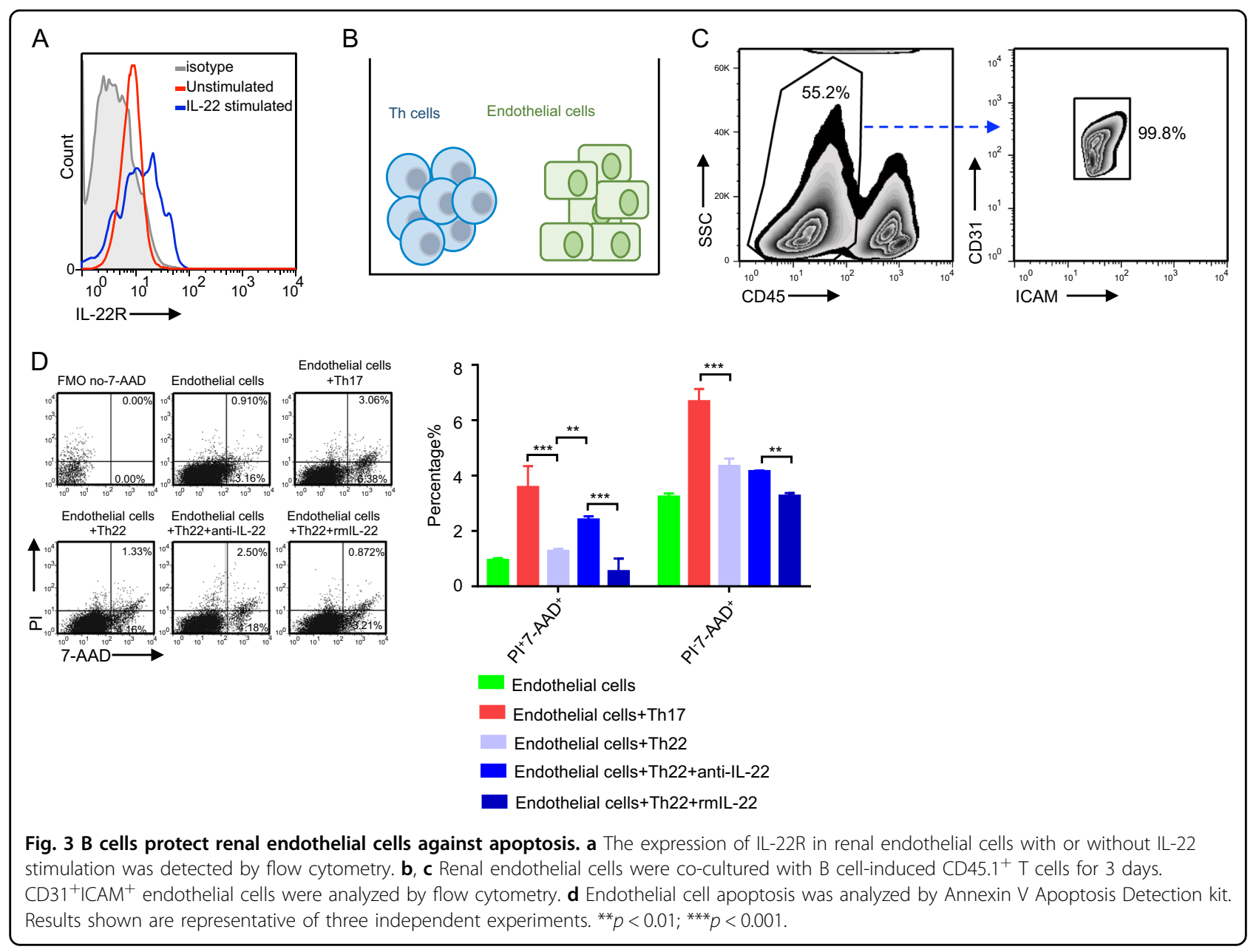

Th22 cells can also play a regulatory role in tissue and cellular repair ${ }^{28,29}$. To analyze B cell-induced Th22 cell function, induced Th22 cells were sorted as described above, then co-cultured with mouse primary renal endothelial cells. Renal endothelial cells highly expressed IL22R and were gated by $\mathrm{CD} 45^{-} \mathrm{CD} 31^{\mathrm{hi}} \mathrm{ICAM}^{\mathrm{hi}}$ (Fig. 3a-c). After 3 days of co-culture, we detected endothelial cell apoptosis, showing that early and late apoptosis were increased when co-cultured with Th17 cells and decreased to control level when cultured with B cellinduced Th22 cells. These effects could be reversed by treatment with anti-IL-22 antibodies in culture media and restored with recombinant mouse (rm) IL-22 (Fig. 3d). These data suggest that activated B cell-induced Th22 cells have protective capabilities over renal endothelial cells.

\section{Activated B cell-induced Th22 cells rescue lupus in vivo}

In all, $10 \times 10^{6}$ ex vivo-activated B cells or PBS control was injected intravenously into MRL/lpr mice weekly for 4 weeks. Mice injected with B cells displayed an obvious reduction in serum titers of anti-ds-DNA antibody, IgM and IgG, and decreased 24-h urine protein levels (Fig. 4a-e). Treatment with activated B cells relieved lupus autoimmune injuries as observed through reduced kidney inflammatory injuries, decreased renal scores, and the depressed weights of spleens and lymph nodes (Fig. $4 \mathrm{f}-\mathrm{h}$ ), whereas activated B cells injected with anti-IL-22 antibodies abolished these therapeutic effects indicating that activated $B$ cells may exert therapeutic effects via IL-22 (Fig. 4a-h).

Furthermore, treatment with activated B cells inhibited the percentage of $\mathrm{CD} 4^{+} \mathrm{IL}-17^{+}$Th17 cells, decreased intracellular RORyt expression in $\mathrm{CD}_{4}{ }^{+} \mathrm{T}$ cells of lupus mice spleens, and reduced serum IL-17. However, it also promoted the differentiation of $\mathrm{CD} 4{ }^{+} \mathrm{IL}-22^{+}$Th2 22 cells, increased serum IL-22, and inhibited intracellular c-Maf expression in $\mathrm{CD}^{+} \mathrm{T}$ cells (Fig. $4 \mathrm{i}-\mathrm{n}$ ). Interestingly, antiIL-22 antibodies injected with B cells reversed B cell regulatory effects on Th17 and Th22 cell differentiation (Fig. $4 \mathrm{i}-\mathrm{n}$ ). These data show that activated B cells could inhibit Th17 but promote Th22 cell differentiation in vivo in lupus-prone mice, supporting the notion that activated B cells could be a promising therapeutic method for treating lupus autoimmunity via IL-22. 


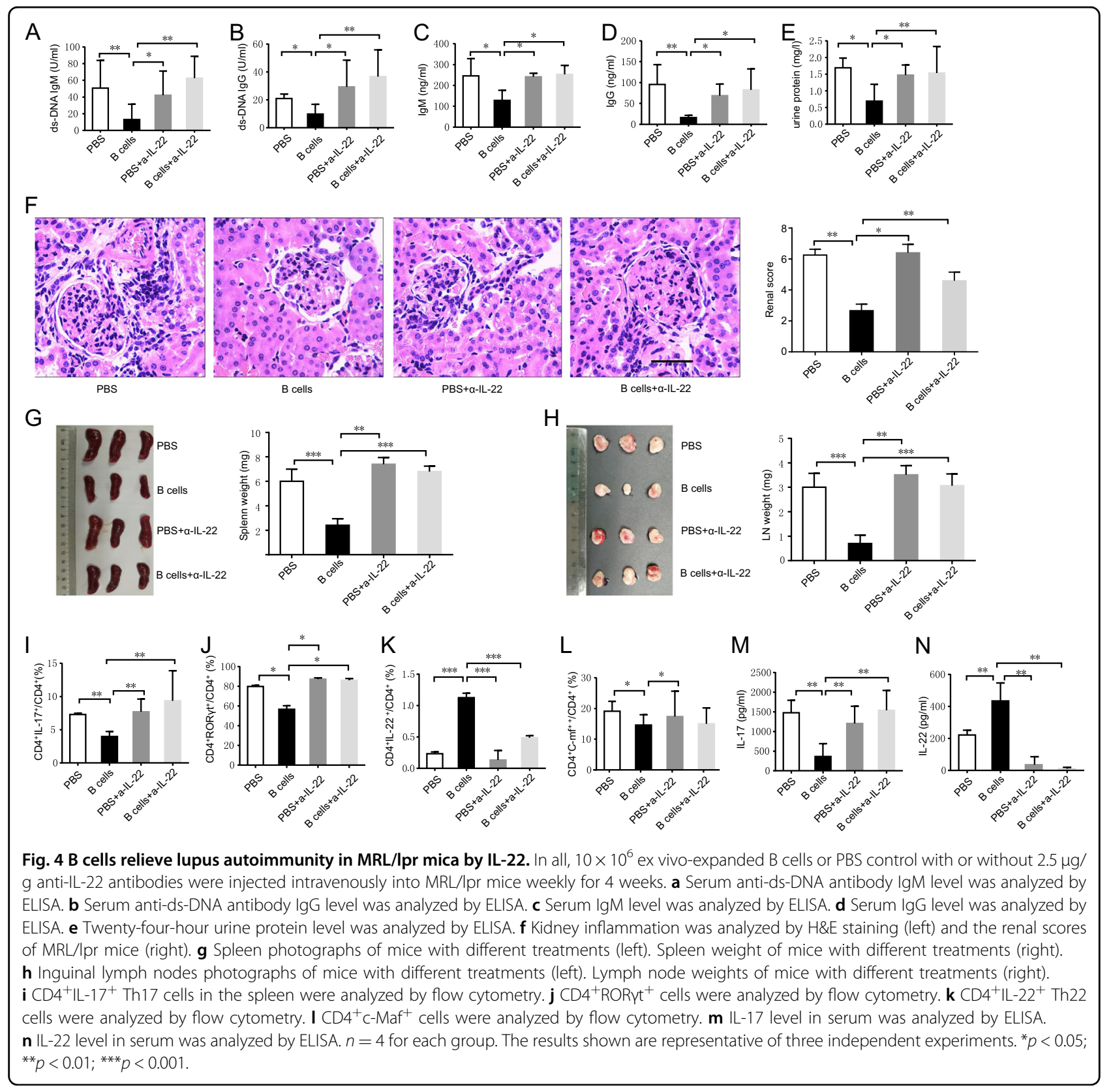

\section{Activated B cells regulate Th17/Th22 differentiation via TNF-a}

We next explored the mechanism through which activated B cells regulate Th22 differentiation. To identify key factors, activated B cells were co-cultured with naive $\mathrm{T}$ cells in different transwell chambers for 5 days. B cells inhibited $\mathrm{CD}_{4}^{+} \mathrm{IL}-17^{+}$Th17 cell differentiation, IL-17 secretion, and RORyt expression (Fig. 5a-d) while promoting $\mathrm{CD}^{+}{ }^{+} \mathrm{IL}_{-} 22^{+}$Th22 cell differentiation, IL-22 secretion, and inhibiting c-Maf expression (Fig. 5e-g). These data indicate that activated $\mathrm{B}$ cells likely inhibit Th17 but promote Th22 cell differentiation without cell contact. We then analyzed the production of cytokines and antibodies produced by activated B cells. Supplementary Fig. 2 shows that $\alpha$-IgM and $\alpha-C D 40$ induced $B$ cell production of large quantities of TNF- $\alpha$, IL-10, TGF$\beta$, IFN- $\gamma$, and IgM and small quantities of IL-4, IL-6, IgG, and IgA (Fig. S2a-c). In addition to previous studies showing that Th22 cells could be derived from naive $\mathrm{T}$ cells in the presence of TNF- $\alpha$ and IL- $6^{22}$, our data demonstrate that B cells also produce TNF- $\alpha$ and other cytokines (Fig. S2a, b). Thus we speculate that B cells might affect Th17/Th22 cell differentiation via TNF- $\alpha$. We next co-cultured B cells with naive T cells under Th17 cell culture conditions with or without anti-IL-4, anti-IL6 , anti-IL-10, anti-TGF- $\beta$, anti-IFN- $\gamma$, or anti-TNF- $\alpha$ 


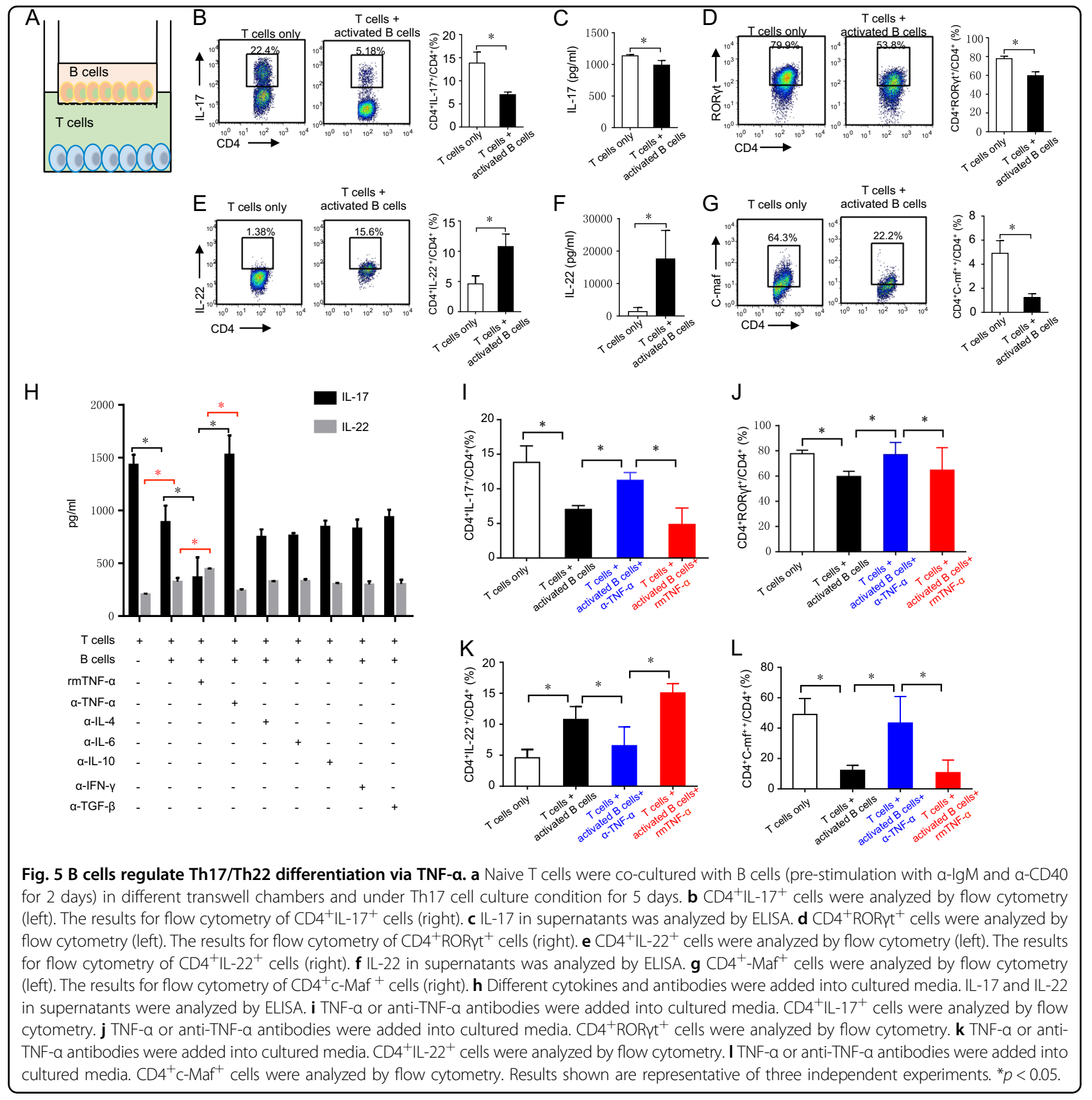

antibodies or rmTNF- $\alpha$. The results illustrate that only rmTNF- $\alpha$ and anti-TNF- $\alpha$ antibodies affected IL-17 and IL-22 production (Fig. 5h). Furthermore, neutralization of TNF- $\alpha$ by anti-TNF- $\alpha$ antibodies inhibited Th22 cell differentiation and IL-22 production but promoted Th17 cell differentiation and IL-17 production. Supplementation with rmTNF- $\alpha$ reversed Th17/Th22 cell differentiation and IL-17/IL-22 production, respectively (Fig. $5 \mathrm{i}-\mathrm{l}$ ). All together, these data indicate that activated B cells may affect Th17 and Th22 cell differentiation via non-contact TNF- $\alpha$ secretion.

\section{Activated $B$ cells regulate $T$ cell differentiation through activation of mTOR}

Although B cells induced Th22 cell differentiation via TNF- $\alpha$, the biological mechanism remained unclear. mTOR activation has been associated with $\mathrm{T}$ cell differentiation ${ }^{30-33}$. Exploring gene expression data in Gene Expression Omnibus, GSE89133 showed that human Th17 cells treated with TNF family member TL1A induced IL-22 secretion, with mTOR activator LAMTOR5 upregulated in the TL1A treatment group after 72-h stimulation ${ }^{24}$ (Supplementary Fig. 3). These data indicated that mTOR 


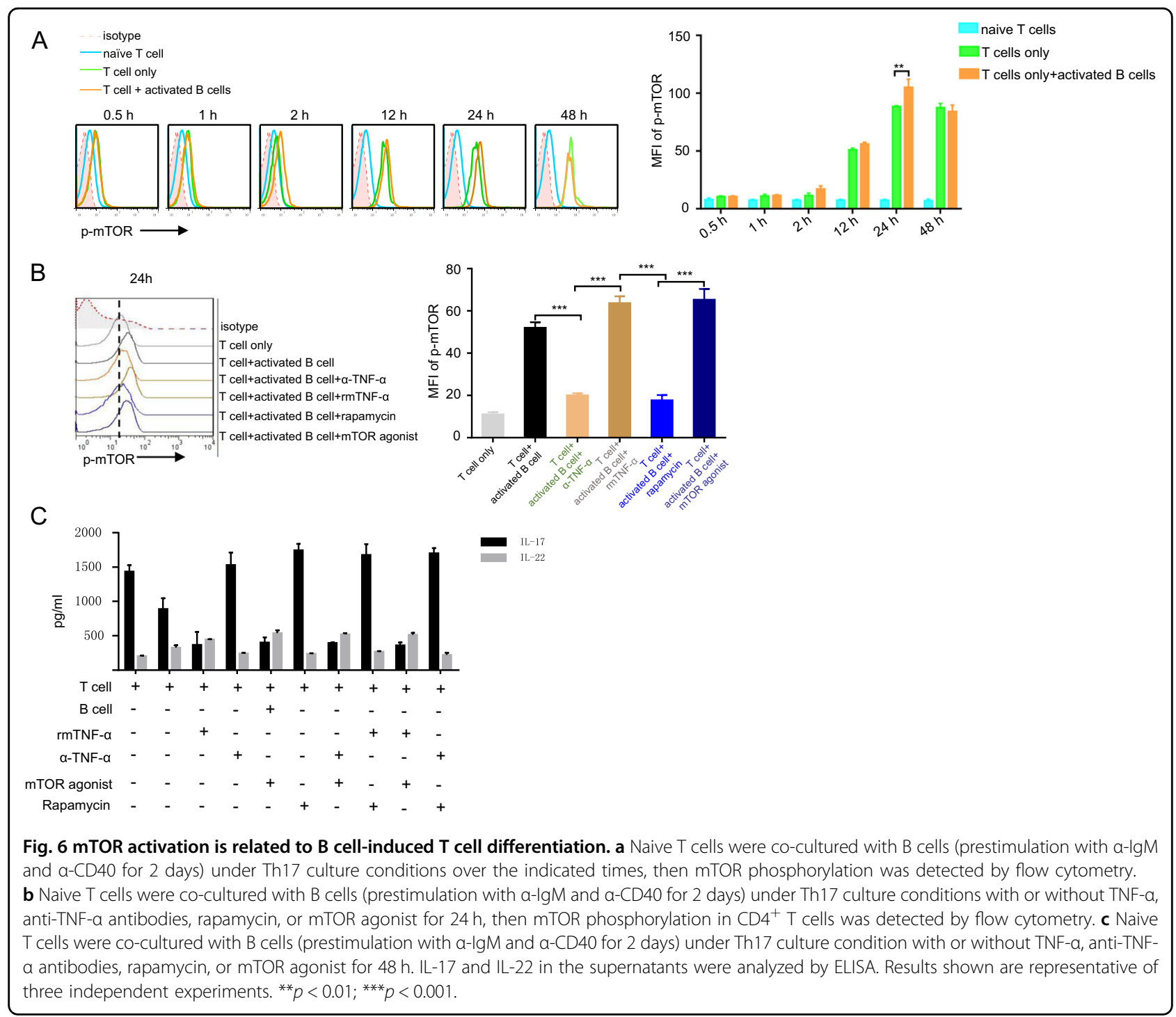

activation might be involved in Th22 cell differentiation. To verify this, naive $\mathrm{T}$ cells were co-cultured with $\mathrm{B}$ cells under Th17 culture conditions over time. Phosphorylation of mTOR was detected across multiple time points in $\mathrm{T}$ cells co-cultured with B cells (Fig. 6a). To further analyze the role of mTOR during $\mathrm{T}$ cell differentiation, naive $T$ cells were co-cultured with $B$ cells under Th17 cell culture conditions with or without the mTOR agonist MHY1485, mTOR inhibitor rapamycin, TNF- $\alpha$, or antiTNF- $\alpha$ antibodies. B cells induced mTOR phosphorylation in T cells, TNF- $\alpha$ and MHY1485 promoted mTOR phosphorylation, and anti-TNF- $\alpha$ antibodies and rapamycin abolished B cell-mediated mTOR phosphorylation (Fig. 6b). Furthermore, when naive $\mathrm{T}$ cells were cocultured with B cells under Th17 cell culture conditions, MHY1485, together with TNF- $\alpha$, promoted IL-22 production but inhibited IL-17 production, whereas rapamycin and anti-TNF- $\alpha$ antibodies abrogated TNF- $\alpha-$ mediated IL-22 production (Fig. 6c). These data suggest that B cells may promote Th22 cell differentiation through TNF- $\alpha$ and mTOR activation.

\section{Activated B cells regulate Th17/Th22 cell differentiation in humans}

To further verify the regulatory effects of B cells on Th17 and Th22 cell differentiation in humans, naive $\mathrm{T}$ cells isolated from PBMCs were co-cultured with B cells for 5 days (naive $B$ cells were prestimulated with $\alpha$-IgM and $\alpha$-CD40 for 2 days). B cells inhibited $\mathrm{CD} 4^{+} \mathrm{IL}-17^{+}$ Th17 cell differentiation and intracellular ROR $\gamma \mathrm{t}$ expression (Fig. 7a, b). Meanwhile, B cells promoted CD4 ${ }^{+} \mathrm{IL}^{-}$ $22^{+}$Th22 cell differentiation but inhibited c-Maf expression (Fig. 7c, d). B cells also inhibited IL-17 secretion but promoted IL-22 production (Fig. 7e, f). Activated B cells also produced large amounts of TNF- $\alpha$ (Fig. 7g, h). In addition, mTOR phosphorylation was upregulated in 


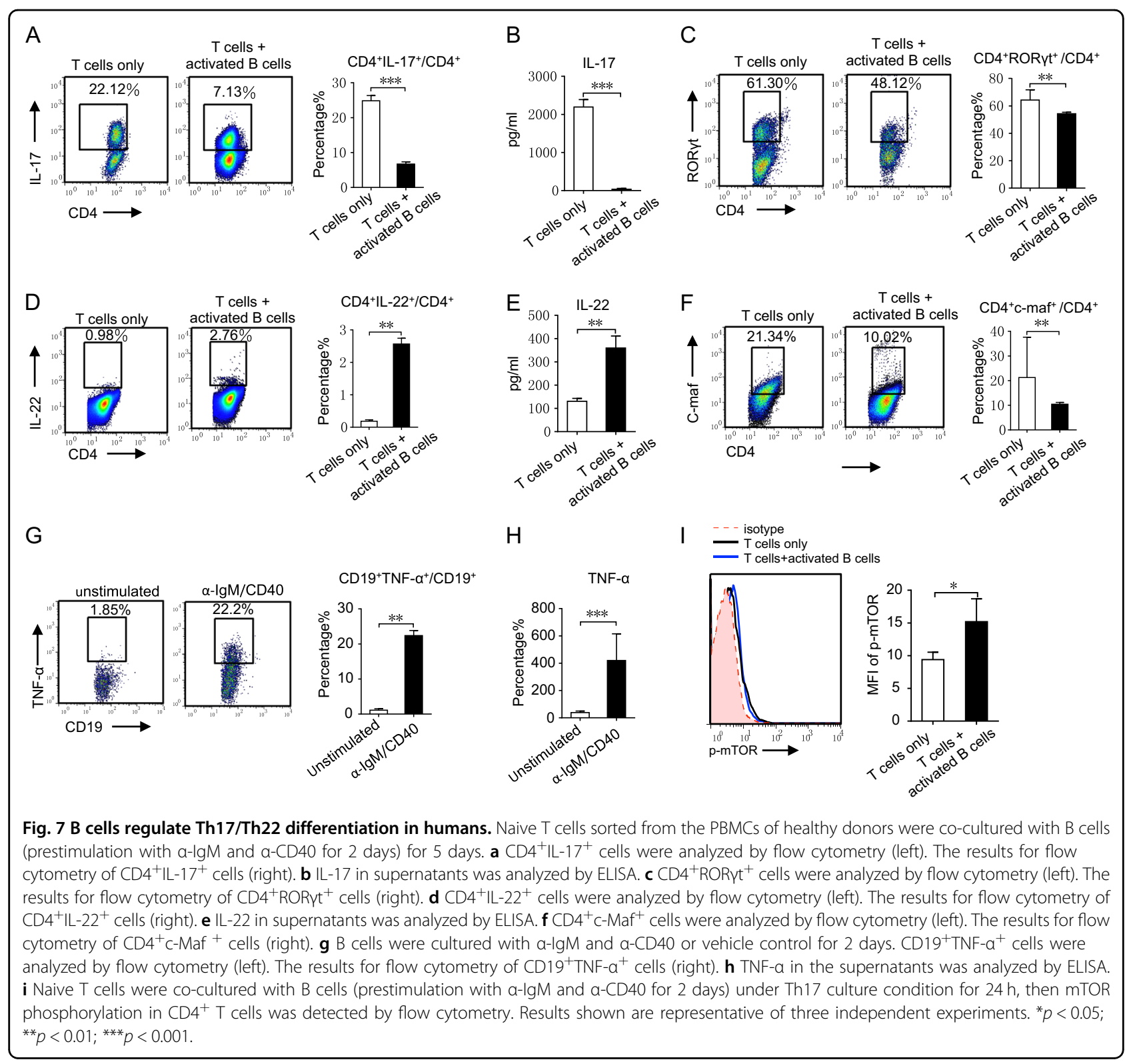

T cells co-cultured with B cells (Fig. 7i). These data indicate that B cells could also affect Th17/Th22 cell differentiation in humans.

\section{Discussion}

B cells possess important immunosuppressive effects and play key negative regulatory roles in many autoimmune diseases ${ }^{3,4,7-9,26}$. Previous studies suggest that B cells play a protective role in the autoimmune inflammation injuries of lupus mice ${ }^{14,34}$. However, the mechanisms of its immunosuppressive effects and how B cells alleviate lupus were not clear. In this study, we found that $\alpha$-IgM- and $\alpha$-CD40-activated B cells co-cultured with Th17 cells in different transwell chambers and supplemented with rmTNF- $\alpha$ promoted IL-22 and inhibited IL-17 secretion, while blocking TNF- $\alpha$ with TNF- $\alpha$-neutralizing antibodies had the opposite effects. However, blocking other cytokines did not achieve similar results. Further investigation showed that TNF- $\alpha$ supplementation promoted Th22 and inhibited Th17 cell differentiation, while blocking TNF- $\alpha$ had the opposite effect.

Th17 cells, under investigation for many years, have proven to play important roles in the pathogenesis of autoimmune diseases and become promising therapeutic targets $^{17-19}$. Antibodies against IL-17 secreted by Th17 cells are marketed clinically to treat psoriatic arthritis and ankylosing spondylitis ${ }^{35,36}$. Th17 cells also play key roles 
in the pathogenesis of lupus; therefore, Th17 cell inhibition may help to alleviate lupus injuries. Here our study demonstrates that activated B cells effectively inhibited Th17 cell differentiation and IL-17 secretion in vitro. In addition, activated $\mathrm{B}$ cell infusion therapy also inhibited Th17 cell expansion in lupus mice. Interestingly, the activated B cells inhibited Th17 cell differentiation while promoting Th22 cell differentiation. Activated B cells regulated Th17 and Th22 cell differentiation only when added to the primary culture stage of naive $\mathrm{CD} 4^{+} \mathrm{T}$ cells; they could not regulate Th17 and Th22 cells when naive $\mathrm{CD} 4{ }^{+} \mathrm{T}$ cells were induced to Th17 cells for 3 days. These results suggest that activated $B$ cells can inhibit naive $\mathrm{CD}^{+} \mathrm{T}$ cell differentiation into Th17 cells and promote the differentiation into Th22 cells.

mTOR activation plays an important role in lymphocyte activation and proliferation ${ }^{30-33}$. In this study, we found that mTOR was activated in $\mathrm{T}$ cells co-cultured with $\mathrm{B}$ cells. TNF- $\alpha$ further promoted mTOR activation and induced IL-22 secretion, while TNF- $\alpha$-neutralizing antibodies inhibited these processes. Since it has been suggested that mTOR activation could be involved in IL-22 secretion from $\mathrm{T}$ cells, we confirmed that an mTOR agonist promoted IL-22 secretion, while rapamycin, an mTOR inhibitor, inhibited IL-22 secretion. These data suggest that mTOR activation might be involved in the differentiation of $\mathrm{T}$ cells into Th22 cells.

Th22 cells are currently considered to have immunosuppressive effects and play negative regulatory roles in psoriasis, arthritis, and hepatitis ${ }^{22,37}$. Studies have shown that cytokines such as TGF- $\beta$, TNF- $\alpha$, and IL- 6 can induce Th22 cell differentiation ${ }^{22,23}$. In this study, a large number of TNF- $\alpha$ secreted by B cells and TGF- $\beta$ initially added to the culture media might together induce $\mathrm{T}$ cells to differentiate into Th22 cells. Although T cells induced by $B$ cells are not all Th22 cells, in vitro functional studies showed that $\mathrm{T}$ cells induced by $\mathrm{B}$ cells protected endothelial cells against apoptosis, inhibited Th1 cell-related cytokine secretion such as IFN- $\gamma$ and TNF- $\alpha$, and also inhibited the B cell secretion of IgM and IgG. Blocking IL22 with neutralizing IL-22 antibodies alleviated the inhibitory effects of effector $\mathrm{T}$ and $\mathrm{B}$ cells. It is suggested that $\mathrm{T}$ cells induced by activated B cells can play an immunosuppressive role through the secretion of IL-22.

Additional in vivo experiments confirmed that activated B cell infusion could alleviate lupus nephritis, reduce dsDNA antibody titer and $24 \mathrm{~h}$ urinary protein, and inhibit Th17 cell differentiation while promoting Th22 cell differentiation. However, co-injection of B cells with IL-22neutralizing antibodies significantly weakened the immunosuppressive and therapeutic effects of B cells on Th17 cells, and the percentage of Th22 cells in vivo were also significantly reduced. These results suggest that B cell infusions might exert therapeutic effects in the treatment of lupus mice by inhibiting Th17 cells and promoting Th22 cell differentiation, along with IL-22 playing a key role in B cell protection.

In summary, we define a novel immunoregulatory role for B cells by inhibiting Th17 and promoting Th22 cell differentiation. B cell infusions effectively relieved lupus autoimmunity injuries, suggesting that $B$ cells could be used as an effective therapy in the treatment of lupus.

\begin{abstract}
Author details
'Department of Dermatology, Zhongshan Hospital, Fudan University, Shanghai, China. ${ }^{2}$ Division of Rheumatology, Huashan Hospital, Fudan University, Shanghai, China. ${ }^{3}$ Institute of Rheumatology, Immunology and Allergy, Fudan University, Shanghai, China. ${ }^{4}$ Department of Immunology, Basic Medical School, Fudan University, Shanghai, China
\end{abstract}

Conflict of interest

The authors declare that they have no conflict of interest.

\section{Publisher's note}

Springer Nature remains neutral with regard to jurisdictional claims in published maps and institutional affiliations.

Supplementary Information accompanies this paper at (https://doi.org/ 10.1038/s41419-020-2362-y).

Received: 26 November 2019 Revised: 17 February 2020 Accepted: 17 February 2020

Published online: 03 March 2020

References

1. Tsokos, G. C. Systemic lupus erythematosus. N. Engl. J. Med. 365, 2110-2121 (2011).

2. Yanaba, K, Bouaziz, J. D., Matsushita, T., Tsubata, T. \& Tedder, T. F. The development and function of regulatory B cells expressing IL-10 (B10 cells) requires antigen receptor diversity and TLR signals. J. Immunol. 182, 7459-7472 (2009).

3. Matsushita, T., Yanaba, K, Bouaziz, J. D., Fujimoto, M. \& Tedder, T. F. Regulatory $B$ cells inhibit EAE initiation in mice while other $B$ cells promote disease progression. J. Clin. Invest. 118, 3420-3430 (2008).

4. DiLillo, D. J., Matsushita, T. \& Tedder, T. F. B10 cells and regulatory B cells balance immune responses during inflammation, autoimmunity, and cancer. Ann. NY Acad. Sci. 1183, 38-57 (2010).

5. Tarique, M. et al. Interleukin-10 producing regulatory B cells transformed CD4 $(+)$ CD25(-) into tregs and enhanced regulatory $T$ cells function in human leprosy. Front. Immunol. 9, 1636 (2018).

6. Vadasz, Z., Haj, T., Kessel, A. \& Toubi, E. B-regulatory cells in autoimmunity and immune mediated inflammation. FEBS Lett. 587, 2074-2078 (2013).

7. Kalampokis, I., Yoshizaki, A. \& Tedder, T. F. IL-10-producing regulatory B cells (B10 cells) in autoimmune disease. Arthritis Res. Ther. 15(Suppl 1), S1 (2013).

8. Mauri, C., Gray, D., Mushtaq, N. \& Londei, M. Prevention of arthritis by interleukin 10-producing B cells. J. Exp. Med. 197, 489-501 (2003).

9. Mauri, C. \& Ehrenstein, M. R. The 'short' history of regulatory B cells. Trends Immunol. 29, 34-40 (2008).

10. Menon, M., Blair, P. A., Isenberg, D. A. \& Mauri, C. A regulatory feedback between plasmacytoid dendritic cells and regulatory $B$ cells is aberrant in systemic lupus erythematosus. Immunity 44, 683-697 (2016).

11. Yang, X. et al. T follicular helper cells and regulatory B cells dynamics in systemic lupus erythematosus. PLOS ONE 9, e88441 (2014).

12. Yang, $X$. et al. T follicular helper cells mediate expansion of regulatory $B$ cells via IL-21 in lupus-prone MRL/pr mice. PLoS ONE 8, e62855 (2013).

13. Heinemann, K. et al. Decreased IL-10(+) regulatory B cells (Bregs) in lupus nephritis patients. Scand. J. Rheumatol. 45, 312-316 (2016).

14. Park, M. J. et al. Myeloid-derived suppressor cells induce the expansion of regulatory $B$ cells and ameliorate autoimmunity in the sanroque mouse model of systemic lupus enythematosus. Arthritis Rheumatol. 68, 2717-2727 (2016). 
15. Harrington, L. E. et al. Interleukin 17-producing CD4+ effector T cells develop via a lineage distinct from the T helper type 1 and 2 lineages. Nat. Immunol. $\mathbf{6}$ 1123-1132 (2005).

16. Park, H. et al. A distinct lineage of CD4 T cells regulates tissue inflammation by producing interleukin 17. Nat. Immunol. 6, 1133-1141 (2005).

17. Yang, J. et al. Th17 and natural Treg cell population dynamics in systemic lupus erythematosus. Arthritis Rheumatol. 60, 1472-1483 (2009).

18. Koga, T., Ichinose, K. \& Tsokos, G. C. T cells and IL-17 in lupus nephritis. Clin. Immunol. 185, 95-99 (2017).

19. Stadhouders, R., Lubberts, E. \& Hendriks, R. W. A cellular and molecular view of T helper 17 cell plasticity in autoimmunity. J. Autoimmun. 87, 1-15 (2018).

20. Jakiela, B. et al. Facilitated expansion of Th17 cells in lupus nephritis patients. Clin. Exp. Immunol. 194, 283-294 (2018).

21. Rutz, S. et al. Transcription factor c-Maf mediates the TGF-beta-dependent suppression of IL-22 production in T(H)17 cells. Nat. Immunol. 12, 1238-1245 (2011).

22. Shabgah, A. G., Navashenaq, J. G., Shabgah, O. G., Mohammadi, H. \& Sahebkar, A. Interleukin-22 in human inflammatory diseases and viral infections. Autoimmun. Rev. 16, 1209-1218 (2017).

23. Basu, R. et al. Th22 cells are an important source of $\mathrm{IL}-22$ for host protection against enteropathogenic bacteria. Immunity 37, 1061-1075 (2012).

24. Thomas, L. S. et al. The TNF family member TL1A induces IL-22 secretion in committed human Th17 cells via IL-9 induction. J. Leukoc. Biol. 101, 727-737 (2017).

25. Plank, M. W. et al. Th22 cells form a distinct Th lineage from Th17 cells in vitro with unique transcriptional properties and Tbet-dependent Th1 plasticity. J. Immunol. 198, 2182-2190 (2017)
26. Bird, A. K. Meednu, N. \& Anolik, J. H. New insights into B cell biology in systemic lupus erythematosus and Sjogren's syndrome. Curr. Opin. Rheumatol. 27, 461-467 (2015).

27. Ivanov, I. I. et al. The orphan nuclear receptor RORgammat directs the differentiation program of proinflammatory $\| \mathrm{L}-17+\mathrm{T}$ helper cells. Cell 126, 1121-1133 (2006)

28. Qin, S. et al. Th22 cells are associated with hepatocellular carcinoma development and progression. Chin. J. Cancer Res. 26, 135-141 (2014).

29. Eyerich, S. et al. Th22 cells represent a distinct human T cell subset involved in epidermal immunity and remodeling. J. Clin. Invest. 119, 3573-3585 (2009).

30. Yang, J. et al. Critical roles of mTOR Complex 1 and 2 for T follicular helper cell differentiation and germinal center responses. Elife 5, e17936 (2016).

31. Perl, A. Activation of mTOR (mechanistic target of rapamycin) in rheumatic diseases. Nat. Rev. Rheumatol. 12, 169-182 (2016).

32. Zeng, $\mathrm{H}$. et al. mTORC1 and mTORC2 kinase signaling and glucose metabolism drive follicular helper $\mathrm{T}$ cell differentiation. Immunity 45, 540-554 (2016).

33. Nagai, S., Kurebayashi, Y. \& Koyasu, S. Role of PI3K Akt and mTOR complexes in Th17 cell differentiation. Ann. NY Acad. Sci. 1280, 30-34 (2013).

34. Watanabe, R. et al. Regulatory B cells (B10 cells) have a suppressive role in murine lupus: CD19 and B10 cell deficiency exacerbates systemic autoimmunity. J. Immunol. 184, 4801-4809 (2010).

35. Mease, P. J. et al. Brodalumab, an anti-IL17RA monoclonal antibody, in psoriatic arthritis. N. Engl. J. Med. 370, 2295-2306 (2014).

36. Reich, K. Anti-interleukin-17 monoclonal antibody ixekizumab in psoriasis. $N$ Engl. J. Med. 367, 274-275 (2012).

37. Cobleigh, M. A. \& Robek, M. D. Protective and pathological properties of IL-22 in liver disease: implications for viral hepatitis. Am. J. Pathol. 182, 21-28 (2013). 